Engineering, Technology and Techniques

Vol.59: e16161011, January-December 2016 http://dx.doi.org/10.1590/1678-4324-2016161011 ISSN 1678-4324 Online Edition

\title{
Energy Efficient Clustering in Multi-hop Wireless Sensor Networks Using Differential Evolutionary MOPSO
}

\author{
D. Rajendra Prasad ${ }^{1 *}$, P. V. Naganjaneyulu ${ }^{2}$, K. Satya Prasad ${ }^{3}$. \\ ${ }^{1}$ Associate Professor, Dept. of ECE, St. Ann's College of Engineering \& Technology; ${ }^{2}$ Principal, MVR College of \\ Engineering \& Technology, Vijayawada; ${ }^{3}$ Professor, Dept. of ECE, Jawaharlal Nehru Technological University, \\ Kakinada.
}

\begin{abstract}
The primary challenge in organizing sensor networks is energy efficacy. This requisite for energy efficacy is because sensor nodes capacities are limited and replacing them is not viable. This restriction further decreases network lifetime. Node lifetime varies depending on the requisites expected of its battery. Hence, primary element in constructing sensor networks is resilience to deal with decreasing lifetime of all sensor nodes. Various network infrastructures as well as their routing protocols for reduction of power utilization as well as to prolong network lifetime are studied. After analysis, it is observed that network constructions that depend on clustering are the most effective methods in terms of power utilization. Clustering divides networks into inter-related clusters such that every cluster has several sensor nodes with a Cluster Head (CH) at its head. Sensor gathered information is transmitted to data processing centers through $\mathrm{CH}$ hierarchy in clustered environments. The current study utilizes Multi-Objective Particle Swarm Optimization (MOPSO)-Differential Evolution (DE) (MOPSO-DE) technique for optimizing clustering.
\end{abstract}

Key words: Wireless Sensor Network (WSN), Clustering, Low Energy Adaptive Clustering Protocol (LEACH), Particle Swarm Optimization (PSO), Multi-Objective Particle Swarm Optimization (MOPSO), Differential Evolution (DE)

\footnotetext{
*Author for correspondence: rp.devathoti@gmail.com
} 


\section{INTRODUCTION}

Wireless Sensor Networks comprise sensor nodes which are arbitrarily distributed in vast areas, gathering necessary data from their environments. Sensor nodes possess restricted amount of power and so, power consuming operations like data gathering, transmissions as well as reception are to be as minimal as possible. Furthermore, it is not possible to carry out replacement or recharging of sensors that are placed in remote or challenging environments. Sensor networks are to send the collected information to Base Stations (BS) or sinks, mostly positioned away from the sensor network environment. Network lifetime therefore is a significant measure of sensor networks' efficacy. Network lifetime denotes the time from the creation to the time when networks become non-functioning (which is usually when even one node dies) [6]. Furthermore, it is vastly agreed upon that balance of power utilization amongst the network nodes is significant in the extension of network lifecycle.

Several problems in wireless sensor networks like node deployment, localization, energy-aware clustering as well as data collection are typically considered as optimization issues. Conventional analytic optimization methods need great amounts of computational effort that rises in an exponential manner when problem size rises. Optimization techniques which need lesser amount of memory as well as computation resources, while concurrently yielding excellent outcomes are favourable, particularly for implementing on single sensor nodes. Optimization techniques that owe their inspiration to nature are effective in terms of computational efforts when compared to analytic techniques.

Clustering refers to the procedure of splitting dataset into subsets called clusters so that information in all subsets shares certain common characteristics. Research into clustering has been happening for a long time. Node clustering protocols are typically carried out in two stages: node clustering set up as well as maintenance. In the former, $\mathrm{CHs}$ are chosen amongst the network nodes. Once $\mathrm{CH}$ is chosen, other nodes associated with the $\mathrm{CHs}$ begin forming the clusters. Nodes which are not $\mathrm{CHs}$ are known as ordinary, member nodes. Clustering has several benefits. Once clustering is complete, gathered information is transmitted to data sinks and this decreases unnecessary as well as repetitive information transfers. It also decreases the quantity of nodes which take part in transmissions and thereby assist in useful power utilization. Moreover, it permits scalability for vast numbers of nodes, decreases transmissions overheads as well as enables effective usage of resources in wireless sensor networks [3].

Because clustering methods lead to decrease in transmission overhead as well as effective resources allotment, they lead to decrease in total power utilization as well as reduction in interferences amongst sensor nodes. Huge amounts of small-sized clusters lead to congestions with while less quantity of clusters drain the $\mathrm{CH}$ with huge quantities of messages communicated from member nodes. Low Energy Adaptive Clustering Hierarchy (LEACH) is a hierarchical routing algorithm on the basis of clustering as well as discovery of optimum quantity of clusters in WSNs for preserving energy as well as enhancing network lifetimes [4].

The primary notion of LEACH is the division of the entire wireless sensor network into many clusters. $\mathrm{CH}$ node is arbitrarily chosen, that is to say, the probability of all nodes to be chosen as $\mathrm{CH}$ is the same. Thereby, power utilization of the entire network is made average, which is how LEACH prolongs network lifetime. 
LEACH protocol is cyclical, that is to say, it functions with several rounds. All rounds comprise two phases: setup as well as steady states. In the former, clusters are formed in a self-adaptive mode and in the latter, information is transferred. The time for the latter stage is typically longer than the first for preserving protocol payloads. The choosing of $\mathrm{CH}$ relies on decisions made 0 as well as 1 . If number is lesser than threshold, nodes become $\mathrm{CH}$ for the current iteration. Threshold is given in equation (1):

$T(n)=\left\{\begin{array}{cl}\frac{p}{1-p^{*}\left(r^{*} \bmod 1 / p\right)} & \text { if } n \in G \\ 0 & \text { else }\end{array}\right.$

Wherein $\mathrm{P}$ refers to the favoured percentage of $\mathrm{CHs}$, $\mathrm{r}$ refers to current iteration while $\mathrm{G}$ refers to the group of nodes which have not been $\mathrm{CHs}$ in the previous $1 / \mathrm{p}$ iterations [8].

Through usage of this threshold, all nodes become CHs at a point within $1 / \mathrm{p}$ iterations. Nodes which have been $\mathrm{CHs}$ are not capable of becoming $\mathrm{CHs}$ for the second time for a minimum of $1 / \mathrm{p}-1$ iterations. After that, all nodes have a $1 / \mathrm{p}$ probability of becoming the $\mathrm{CH}$ in each iteration. When an iteration is over, all member nodes which are not $\mathrm{CHs}$ choose closest $\mathrm{CH}$ and join the cluster for forwarding data. CHs combine and aggregate the data and transmit it to BSs, thereby extending lifetime of major nodes.

The node chosen as cluster head forwards a message through the CSMA MAC protocol while non-cluster head nodes select closest cluster head and join its cluster. Figure 1 illustrates LEACH's clustering structure.

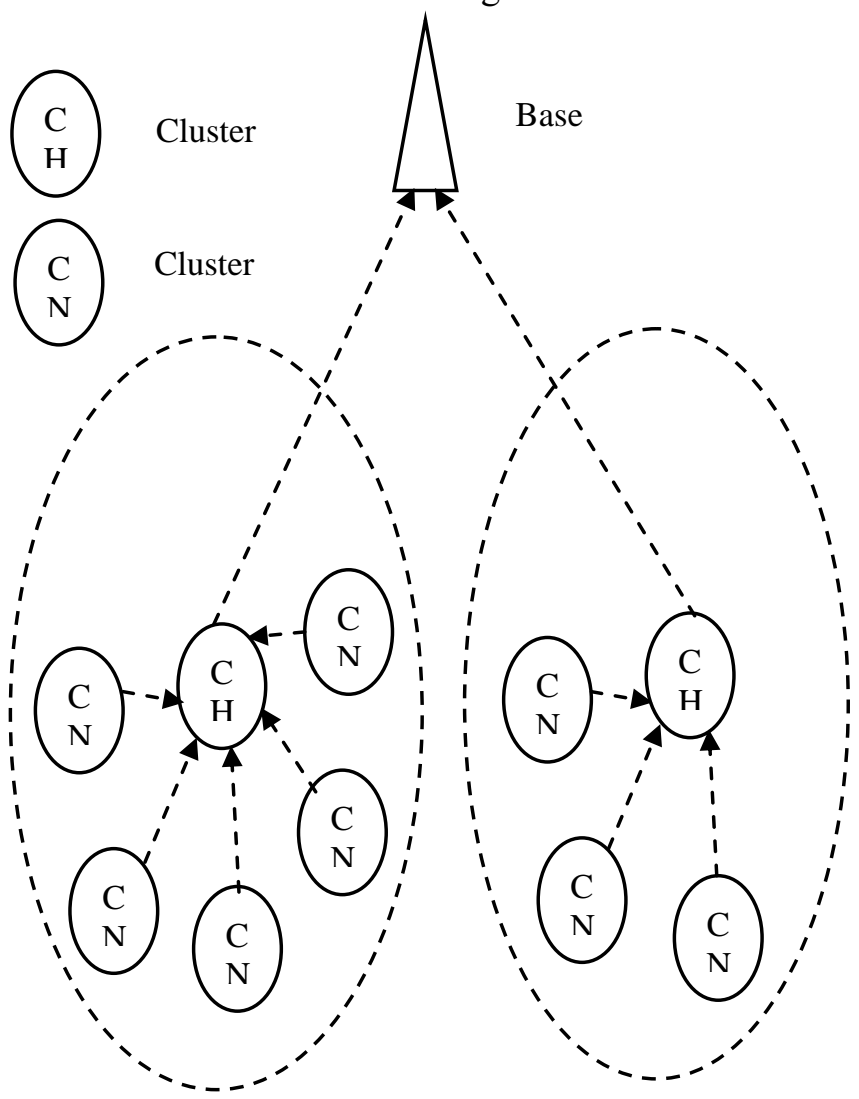

Figure1- Cluster formation in LEACH

Cluster heads now create TDMA schedules for all nodes it their clusters for transmitting data. Every member node of the cluster forwards data to the cluster 
head as per a particular schedule. In the end, every cluster head performs aggregation of the data and forwards it to base stations.

Cluster Formation Protocol [21]:

Step1: CHs broadcast advertisement messages (ADV) through CSMA MAC protocol

Step 2: On the basis of the Received Signal Strength of ADV messages, all non-CH nodes determine their $\mathrm{CH}$ for the current cycle (arbitrary selection with obstacle).

Step 3: Every non-CH forwards join-request message (Join REQ) to the Selected $\mathrm{CH}$ utilizing CSMA MAC protocol

Join-REQ = node ID + CH ID + header

Step 4: CH initialize TDMA schedule for data forwarding coordination with in the cluster.

Step 5: TDMA Schedule avoids collision amongst data messages as well as energy preservation in non $\mathrm{CH}$ nodes.

Low-power optimization methods built for traditional ad-hoc networks are not adequate because they do not appropriately address certain characteristics of embedded as well as sensor networks. It is not sufficient to decrease total power utilization, it is also necessary to make maximum the network lifetime, i.e. maintain entire network connectivity for the maximal amount of time.

Energy efficacy, costs as well as application requisites are the obstacles to be handled when constructing wireless sensor networks. It needs optimization of hardware as well as software for making wireless sensor networks effective. Software handles the problem of network lifetime. Many optimization protocols are available for various issues and the selection of the right protocol is significant in resolving the issue.

In this paper, energy efficient LEACH, PSO, MOPSO, DE and MOPSO-DE are evaluated for clustering. Section 2 explains the literatures that are related to the study, section 3 explains the methods used, section 4 explain the results and finally section 5 concludes the work.

\section{RELATED WORKS}

Nguyen, et al., [1] discussed novel discoveries on the complexity of $\mathrm{CH}$ selection protocols. Two $\mathrm{CH}$ selection variations are studies: 1) distancerestricted selection herein all network nodes are situated within a particular distance to the closest $\mathrm{CH}$ and 2) size-restricted selection wherein clusters are permitted to have restricted number of member nodes. The technique revealed that the issue of minimization of group of CHs is an NP-hard issue for both variations. The investigators suggested two distributed selection protocols, both with logarithmic approximation ratios for the variations.

Romoozi \& Ebrahimpour-Komleh [2] examined intelligent methods for node situating to decrease power utilization with coverage conserved in wireless sensor networks. Genetic Algorithms were utilized for creating energy effective node situating in wireless sensor networks. Simulations revealed that the suggested intelligent protocol prolonged network lifetime for various network situating techniques.

He et al., [3] suggested a clustering method for balancing energy on the basis of genetic clustering protocol. The novel protocol merges genetic algorithm as well as Fuzzy C-Means (FCM) clustering protocol for overcoming sensitivities of the initial values of FCM. Optimum clusters are created in the network and then CHs are chosen in every cluster. Simulations reveal that when contrasted with LEACH, the protocol balances the energy costs of sensor nodes, prolongs network lifetime effectively and outperforms LEACH. 
Singh \& Lobiyal [4] suggested the PSO method for creating energy-aware clusters through optimum selections of CHs. Particle Swarm Optimization decreases costs of discovering optimum positions for CHs. Additionally, PSO method in the cluster instead of BS has been executed, making it a semidistributed technique. Selection criterion of objective functions have their basis in remaining energy, intra-cluster distances, node degrees as well as head counts of potential CHs. In the end, simulations confirm the efficacy of the suggested method with regard to network lifetime, average packet transmission, $\mathrm{CH}$ selections.

Kumar [5] suggested and tested two novel clustering-based algorithms for heterogeneous wireless sensor networks that are known as Single-Hop EnergyEfficient Clustering Protocol (S-EECP) as well as Multi-Hop Energy-Efficient Clustering Protocol (M-EECP). In the former, $\mathrm{CHs}$ are chosen by weighted probabilities on the basis of ratios between remaining energy in every node as well as average energy of the network. Nodes with great amounts of initial energy as well as remaining energy are more likely to be chosen as CHs than those with lesser energy while in the latter, the chosen $\mathrm{CHs}$ transmitted the information packets to base stations through multi-hop transmission method. Network lifetime was studied and simulations revealed that the suggested algorithm extended network lifetime apart from achieving load balancing amongst CHs.

Elhabyan and Yagoub [6] suggested a PSO-based method for finding optimum quantity of $\mathrm{CHs}$ for maximizing network energy efficacy. Concurrently, network coverage as well as link quality is regarded. Impact of utilizing realistic network as well as energy utilization framework in cluster-based communications for wireless sensor networks was looked into. Simulation reveals that the novel algorithm performed better than other cluster-based algorithms with regard to average energy utilization apart from having adequate information packet throughputs.

Maleki et al., [7] suggested hybrid PSO as well as Differential Evolution (DE) protocols which are metaheuristic protocols and that analyse area coverage issues in WSNs. PSO algorithms are employed for comparing hybrid system's efficacy in like situations. Simulations reveal that the hybrid protocol improved network lifetime apart from making optimized usage of sensor energy through optimization of sensor coverage when contrasted with PSO.

Tyagi \& Kumar [8] suggested energy-aware routing for WSNs. The most famous one for clustering in WSNs is LEACH which has its basis in an adaptive clustering method. The investigators presented the taxonomy of several clustering as well as routing methods in wireless sensor networks on the basis of measures like energy as well as power management, network lifetimes, optimum $\mathrm{CH}$ selections, multi-hop information transmissions and so on. A succinct discussion regarding the several benefits as well as shortcomings of the many algorithms present such that it can be of assistance to researchers in selecting appropriate protocols on the basis of their comparative advantages.

Aziz et al., [9] presented two important problems in mobile wireless sensor networks which are coverage as well as power saving. Excellent coverage rates ensure excellent Quality of Service (QoS) for the WSN. Conserving energy extends network lifetimes. The two problems are correlated because improving coverage in mobile wireless sensor networks needs sensors to travel around, which is a key element in the utilization energy. Hence, optimizing coverage ought to consider the remaining energy. When observing the restricted amount of energy present in the sensors, the authors suggested a PSO-based method for maximization of coverage with a restriction on the maximal distance that sensors may move. Simulations reveal that the suggested protocol attains 
excellent coverage and considerably decreases power utilization for the repositioning of sensors.

Yu \& Xiaohui [10] suggested a clustering algorithm on the basis of PSO which has a balanced perception of distance as well as energy of double $\mathrm{CH}$ centralized networks. It primarily enhances basic PSO through the optimization of fitness functions. Additionally, the protocol's information transfer stage in the main division of labour amongst $\mathrm{CHs}$ for extending the $\mathrm{CH}$ re-selection cycle. Simulations reveal that the protocol managed balanced power utilization across the network while also prolonging network lifetime.

Rostami \& Mottar [11] suggested a novel technique for clustering WSNs on the basis of PSO protocol through usage of optimum fitness functions that aim at prolonging network lifetimes. The variables utilized in the protocol are remaining energy density, distance from BS, intra-cluster distance from $\mathrm{CH}$. Simulations reveal that the suggested technique outperformed other algorithms with regard to network lifetimes as well as power utilization.

Elhabyan\&Yagoub [12] suggested two Linear Programming (LP) designs for the issues of clustering as well as routing as well as two protocols for the same on the basis of PSO. The clustering protocol discovers optimum set of CHs which make maximum the energy efficacy, clusters quality as well as network coverage. Routing protocol was formulated with a new particle encoding strategy as well as fitness functions for finding optimum routing trees which connect the $\mathrm{CHs}$ to the base station. The two protocols are combined into one two-tier algorithm for providing complete as well as practicable clustering algorithm. The impact of utilizing realistic energy utilization framework in cluster-based transmission for wireless sensor network is studied. The outcomes prove that the suggested algorithm outperforms with regard to scalability, Packet Delivery Ratio (PDR) at CHs as well as overall delivery of information packets to base station.

Splitting network into optimum quantity of clusters as well as choosing optimum set of nodes as CHs is an NP-hard issue. NP-hard nature of clustering issues ensures that it is an adequate candidate for applying evolutionary algorithms as well as particle swarm optimization algorithms. Yadav et al., [13] presented a solution on the basis of PSO to the issue of optimum clustering through usage of remaining energy as well as transmission distance of sensor nodes. Simulations reveal that there is significant enhancement in network lifetimes as opposed to other already present protocols.

\section{METHODOLOGY}

In this section, we discussed the PSO and proposed MOPSO algorithm to improve the clustering.

\section{Particle Swarm Optimization (PSO)}

PSO was suggested by Kennedy and Eberhart [14] and it is utilized in the resolution of several issues in several fields, particularly engineering as well as computer science. Particles fly through multi-dimensional search spaces with every particle denoting potential solution to the multi-dimensional optimization issue. All solutions fitnesses have their basis in performance functions associated with the optimization issue being resolved. The motion of the particles is grounded in two elements utilizing from iteration to iteration and particle to particle. Because of the former, particles store the best location visited previously as pbest and because of the latter, particles store the information regarding best location visited by any particle in the swarm as a whole as gbest. Particles are attracted to these best solutions. 
PSO is an evolutionary computational method that has its basis in the social activity of flocks of birds. Swarms refer to the quantity of possible solutions to the optimization issue, wherein all possible solutions are known as particles. The objective of PSO is the discovery of the particle position which leads to best evaluation of specified fitness function. During the procedure of initialization, all particles are arbitrarily assigned initial variables and are sent flying through multi-dimensional search space. In every generation, all particles utilize the data regarding earlier best individual location as well as global best for maximizing the probability of travelling toward better solution space which results in better fitness. When fitness is better than individual best, it substitutes the individual one and its potential solution is updated.

Dimension D of all particles is the same. A particle $P_{i}, 1 \leq i \leq N_{P}$ has position $X_{i d}, 1 \leq d \leq D$ as well as velocity $V_{i d}$ in the $\mathrm{d}^{\text {th }}$ dimension of the hyperspace. It adopts the notation for denoting the $\mathrm{i}^{\text {th }}$ particle $P_{i}$ of the population thus [2]:

$P_{i}=\left[X_{i, 1}, X_{i, 2}, X_{i, 3}, \ldots, X_{i, D}\right]$

All particles are tested by fitness functions for judging quality of solutions to the issue. For reaching global best position, particle $P_{i}$ moves towards Pbest $_{i}$ as well as gbest for updating its own velocity as well as position. In every iteration, velocity $V_{i d}$ as well as position $X_{i d}$ in $\mathrm{d}^{\text {th }}$ dimension is updated through:

$\mathrm{V}_{\mathrm{id}}(\mathrm{t})=\mathrm{w}^{*} \mathrm{~V}_{\mathrm{i}, \mathrm{d}}(\mathrm{t}-1)+\mathrm{c} 1 * \mathrm{r} 1 *\left(\right.$ Xpbest $\left._{\mathrm{i}, \mathrm{d}}-\mathrm{X}_{\mathrm{i}, \mathrm{d}}(\mathrm{t}-1)\right)+\mathrm{c} 2 * \mathrm{r} 2 *\left(\right.$ Xgbest $\left._{\mathrm{d}}-\mathrm{X}_{\mathrm{i}, \mathrm{d}}(\mathrm{t}-1)\right)$ (3)

$$
X_{i, d}(t)=X_{i, d}(t-1)+V_{i, d}(t)
$$

Wherein $\mathrm{w}$ refers to inertial weight, $c_{1}$ as well as $c_{2}$ are two non-negative constants known as acceleration factor while $r_{1}$ as well as $r_{2}$ represent two distinct uniformly distributed arbitrary numbers within [0,1]. The update procedure is iterated till adequate gbest is attained or a specified count of iterations $t_{\max }$ is attained.

Typical single-objective PSO protocol begins with an initialization of the swarm as well as the position/velocity of particles. The respective pbest of all particles are initialized and the leader. During a maximal count of iterations, all particles fly through the search space updating their positions. They are evaluated with pbest also being computed. When every iteration is completed, leaders are updated. Leaders are those particles with gbest or another particle based on the social architecture of the swarm.

\section{Multi-Objective Particle Swarm Optimization (MOPSO)}

Generic Multi-Objective Optimization Problems (MOOP) may be expressed by minimizing function $\mathrm{f}(\mathrm{x})$, subject to $\mathrm{p}$ inequality as well as $\mathrm{q}$ equality restrictions in equation (5):

$$
\begin{aligned}
\min . f(x)= & \left\{f_{1}(x) f_{2}(x) \ldots . . f_{m}(x)\right\}^{T} \\
& x \in D
\end{aligned}
$$

Wherein $x \in R^{n}, f_{i}: R^{n} \rightarrow R$ while $\mathrm{D}$ refers to potential search space; $x=\left\{x_{1} x_{2} \ldots x_{n}\right\}^{T}$ represents the set of $\mathrm{n}$-dimensional decision parameters (continuous, discrete or integer); $\mathrm{R}$ refers to the set of real numbers while $R^{n}$ denotes n-dimensional hyper-plane or space. 
MOOP is to concurrently perform optimization of vector functions as well as production of Pareto optimum solutions. Pareto front refers to a set of Pareto optimum (non-dominated) solutions. A solution $\mathrm{x}^{*}$ is considered as dominated by a solution $\mathrm{x}$, if and only if, $\mathrm{x}$ is equal to or better than $\mathrm{x}^{*}$ with regard to all objectives.

For applying PSO scheme for the resolution of multi-objective optimization issues, the initial strategy is to be altered. When resolving multi-objective issues, three objectives are mandatory: maximizing the quantity of elements in the discovered Pareto optimum set, minimizing the distance of the Pareto fonts yielded by the protocol with regard to the global true Pareto front (presuming the location is known) and maximizing distribution of solutions discovered such that there is a smooth as well as uniform distribution of vectors.

Two primary methods for formulating PSO protocols in the case of multiobjective issues are present. Firstly, there are protocols that regard every objective function in a separate manner. Every particle is tested for one objective function at one time and determining the best position is carried out like in the case of single-objective issue. The primary obstacle in this method is the appropriate manipulation of data arising from every objective function for guiding particles towards Pareto optimum solutions.

Secondly, there are protocols which evaluate all objective functions for every particle, and on the basis of the notion of Pareto optimality, they yield nondominated best positions which are utilized for guiding the particles. Determining the leaders (non-dominated best positions) is not an easy task because there may be several in a particle's neighbourhood, however only one is typically chosen for participation in the velocity update. .

Updating particle's pbest is carried out in the former approach like how it is done in generic PSO for single-objective optimizations. In Pareto-based methods, best positions of particles are substituted by new ones only if they dominate the older. If new as well as earlier best positions are non-dominated, older one is typically substituted for promoting swarm diversity [16]

Typical Multi Objective PSO (MOPSO) therefore begins with initialization of swarm with usage of external archives for storing leaders that are taken from non-dominated particles in swarm. After initialization of leader, archive, certain quality metrics are computed for all leaders to typically choose one leader for every particle of the swarm. In the primary loop of the protocol, movement of all particles are carried out after leader are chosen and may be, mutations or turbulence operators are employed. Particles are then tested and respective pbests are computed. Once every iteration is complete, group of leaders are updated and quality metrics are computed once more. When terminating criteria are met, archive is given as outcome of the search.

\section{Differential Evolution (DE)}

Conventional LEACH presumes that every node holds equal amount of energy. The choosing of cluster heads does not regard the remaining energy left in the nodes but it important to consider this for the optimization of the choosing of cluster heads [17].

$\mathrm{DE}$ is a rising evolutionary protocol and similar to PSO, DE is an optimization protocol that has its basis in the theory of Swarm Intelligence, and performs optimization of searches through the cooperation as well as competition between individuals in the swarm.

DE has a relatively strong global convergence capacity as well as resilience and does not require assistance from knowledge regarding the problem features and hence it is suitable for complex optimization issues.

DE protocol includes [18]: 
Creation of initial population: Initial population (solution vectors) is selected arbitrarily from DE protocol problem domain. The solutions position vector is presented by equation (6).

$$
\begin{aligned}
& X_{i}=\left(\mathrm{x}_{i, 1}, \mathrm{x}_{i, 2}, \ldots ., \mathrm{x}_{i, D}\right) \\
& x_{i k}=x_{k}^{\min }+\operatorname{rand}(0,1) .\left(\mathrm{x}_{k}^{\max }-\mathrm{x}_{k}^{\min }\right) \\
& \text { with } \mathrm{i} \in[1, \mathrm{~Np}], \mathrm{k} \in[1, \mathrm{D}]
\end{aligned}
$$

Selecting random numbers $\mathrm{x}_{\mathrm{ik}}$ from a problem domain is through equation (7). Here, D represents solution dimensions, $\mathrm{Np}$ represents quantity of the initial population. Rand $(0,1)$ function yields uniformly distributed arbitrary numbers between $(0,1)$. It is noted that when the equation (7) is utilized, values for $\mathrm{x}_{\mathrm{ik}} \mathrm{X}$ is in $\left[\mathrm{x}_{i}^{\max }, \mathrm{x}_{i}^{\min }\right]_{\text {as }}$ well as the solutions position vector become a possible solution to the optimization issue.

Mutation: Three vectors $r_{1}, r_{2}$ and $r_{3}$ that are non-equal and located between [1, $\mathrm{Np}$ ] are arbitrarily chosen,. G represents the quantity of generations while $\mathrm{F}=0.5$ is a constant. For $\mathrm{x}$ vector in the population, a novel solution in every iteration is generated as per equation (8).

$$
v_{i, G+1}=x_{r_{1}, G}+F \cdot\left(x_{r_{2}, G}-x_{r_{3}, G}\right)
$$

Crossover: This improves population diversity. Novel vectors are generated through a hybrid of $\mathrm{x}$ and $\mathrm{v}$ vectors like in equation (9).

$$
u_{j i, G+1}= \begin{cases}v_{j i, G+1} & \text { if }\left(r_{j} \leq C R\right) \text { or } j=j_{\text {rand }} \\ x_{j i, G+1} & \text { otherwise }\end{cases}
$$

(9)

Variable CR is situated in $[0,1)$. Variable $r_{j}$ is arbitrarily generated between $[0$, 1] and the value is $j=1,2 \ldots D$.

Selection: For choosing high propriety vectors, vectors generated by mutation as well as crossover operators are contrasted with one another and that which is more appropriate is carried over to the subsequent generation. Selection operation occurs through equation (10).

$$
x_{i, G+1}=\text { Fitness Value }\left(u_{i, G+1}, x_{i, G}\right)
$$

Stop: The search procedure it iterated till terminating condition is fulfilled. Typically, it is based on a constant propriety of best solution to the protocol's iteration.

\section{Proposed Hybrid MOPSO-DE Protocol}

In proposed hybrid DE - PSO algorithm based approach, selection of $\mathrm{CH}$ in MOPSO is attained through fitness functions obtained in an analytic manner wherein transmission energy is regarded as important. Distance between the transmitting elements utilizes energy. Other elements such as remaining energy as well as Energy Constraint (EC) measure are also regarded. If a node possesses great amounts of energy remaining, more neighbours as well as excellent signal strength, it has an excellent change of being chosen as $\mathrm{CH}$. The objective function of $\mathrm{CH}$ is given in equation (11):

$q_{1}=\left(E_{i}\right)^{k_{1}} *\left(K_{i}\right)^{k_{2}} *\left(S E_{i}\right)^{k_{3}}$

Wherein $E_{i}$ denotes remaining energy, $K_{i}$ denotes a set of neighbours, $S E_{i}$ represents signal strength while $k_{1}, k_{2}, k_{3}$ denote weights controlling $E_{i}, K_{i}$ as well as $S E_{i}$. 
$\mathrm{CHs}$ choose routes for transmitting data in a dynamic manner on the basis of path measures such as power utilization. Energy restricted metrics are utilized when looking for several paths between $\mathrm{CHs}$ as well as sink node. EC measure calculates inter-flow interferences as well as communication rate variations as well as wireless link loss ratio. It is given in equation (12):

$$
\operatorname{IEC}_{i j}(c)=\operatorname{ETT}_{i j}(c) *\left|N_{i}(c) \cup N_{j}(c)\right|
$$

Wherein $N_{i}(c)$ represents a set of neighbors of node $I, C$ refers to channel $c$ $\left|N_{i}(c) \cup N_{j}(c)\right|$ denotes total nodes interfered with by communication activity between Node $i$ as well as Node $j$ over channel $c$.

$\operatorname{ETT}_{i j}(c)$, expected transmission time, that calculates communication rate difference as well as link loss ratio.

DE performs better than other optimization protocols with regard to convergence speed as well as resilience across several numerical benchmark functions as well as real world issues as per current research works. Typically, hybridization strategies are sorted into two kinds which are staged pipelining type hybrid and additional-operator type hybrid. In the former, optimization is employed on all individuals in the population after which there is more enhancements utilizing DE. In the latter, DE is employed as a generic genetic operator for a related probability. In this scheme, the former is utilized for its several benefits. As per this technique, all generations after PSO stochastic optimization procedure is employed on all population individuals, choose $n$ best Differential vectors from the current population on the basis of fitness values for generating initial population needed for local search through DE. The search is iterated until it arrives at the maximal quantity of generations or the terminating condition is fulfilled [19].

The suggested MOPSO-DE protocol is as follows [20]:

\section{Step 1: Initialize the particles}

A population of size $\mathrm{N}$ is initialized. Here, a particle's decision parameters are set through equation (13).

$$
\operatorname{rand}(0.0,1.0) *(U B-L B)+L B
$$

Here, rand $(0.0,1.0)$ denotes an arbitrary number initialized uniformly between $[0.0,1.0]$. LB as well as UB represents the Lower-Bound (LB) as well as Upper-Bound (UB) correspondingly of the decision parameters of the MO problem samples. Velocity is set to an arbitrary value between [0, UB - LB]. The personal best of an individual is initialized to the current value of the decision parameter. Half the populations' direction is reversed through initializing of the velocity to negative as per a coin toss to better search the search-space. The particles are then appraised with the objective functions and fitness is designated. The fitness values are utilized for determining dominance. 


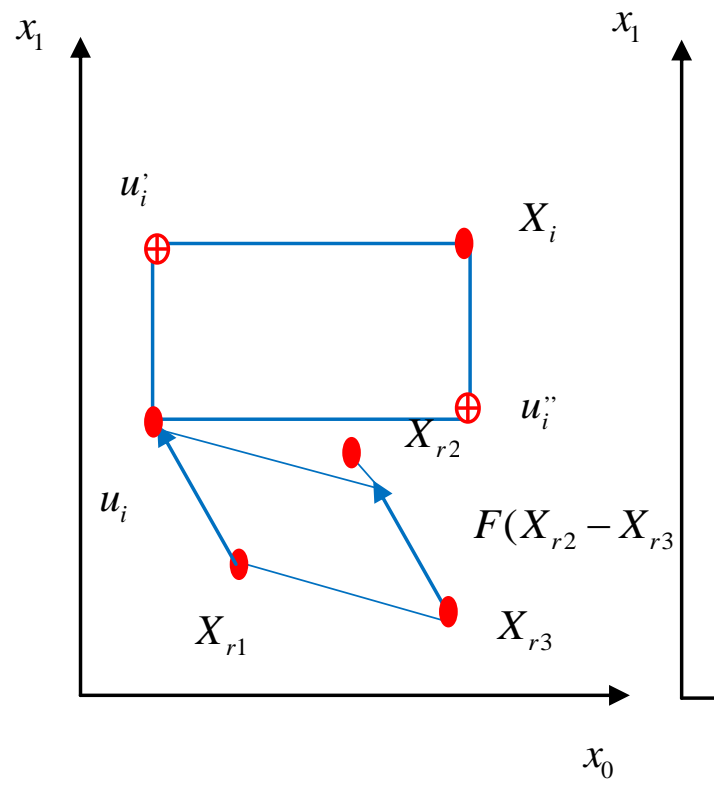

(a)

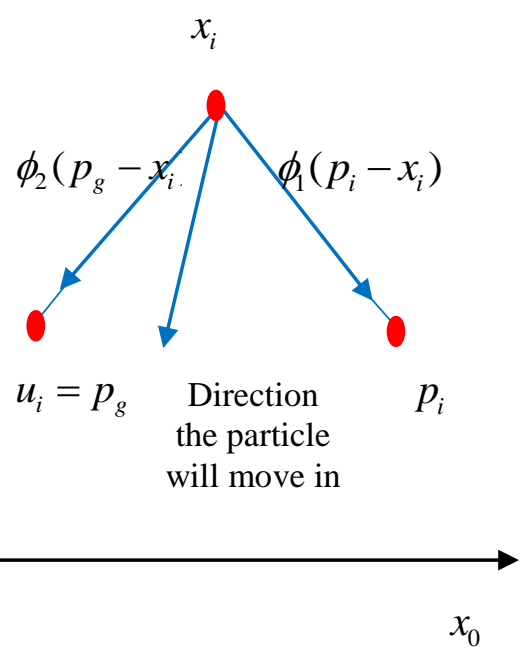

(b)

Figure 2- (a) Creating a leader vector using the DE operator (b) Applying the PSO rules to move the particle

Step 2: Create leaders and move the particles

All particles xi will select three other particles $X_{r 1}, X_{r 2}, X_{r 3}$ from the population so that $i \neq r 1 \neq r 2 \neq r 3$. Then a leader vector $\left(u_{i}=p_{g}\right)$ is obtained from the DE operator in equation (9). All particles will move toward their leader vectors (figure 2)

\section{Step 3: Update the particles' personal bests}

The particles are appraised as per the objective functions and fitness is designated. Next, the particles' personal bests are updated as per their current positions and best positions discovered as of yet.

updating their velocities and positions as per the PSO rules in equations (3) and (4).

Step 4: Obtain the particles for moving to the next iterationThe population of $\mathrm{N}$ particles at the start of the iteration is fused with the $\mathrm{N}$ quantity of particles which have altered their positions

for creating a population of size $2 \mathrm{~N}$. The non-dominated sorting procedure is employed on this $2 \mathrm{~N}$ population for obtaining $\mathrm{N}$ particles that are carried over to the subsequent cycle. The steps 2 to 4 are iterated till the maximal quantity of iterations is arrived at.

\section{RESULTS \& DISCUSSION}

The evaluation setup comprises varying quantity of sensor nodes (75 to 450) and one sink spread over an area of $4 \mathrm{sq} . \mathrm{Km}$. The simulations are run for 300 sec. The proposed MOPSO-DE is compared with LEACH, PSO and MOPSO. Parameters like average end to end delay, average PDR and the percentage of 
nodes alive is measured during the simulations. Table 1 displays the simulation parameters utilized.

Table 1 - Simulation Parameters Used

\begin{tabular}{|l|l|}
\hline Parameter & Value \\
\hline Number of particles & 20 \\
\hline Inertia weight (min-max) & $0.2-0.9$ \\
\hline Velocity (min-max) & $1-5$ \\
\hline Constants (c1 \& c2) & 2 \\
\hline
\end{tabular}

Table 2 - Average End to End Delay (sec)

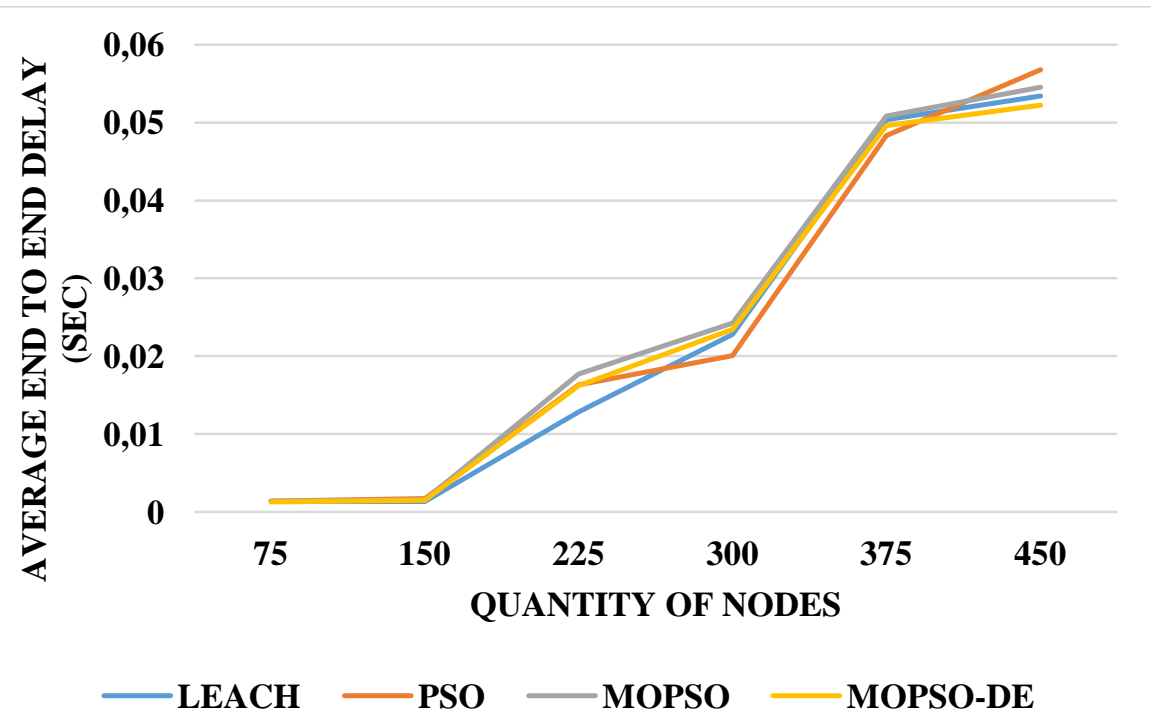

Figure 3 - Average End to End Delay (sec)

From the figure 3, it is seen that the MOPSO-DE has lower average end to end delay by $2.98 \%, 23.14 \% \& 1.55 \%$ for LEACH, by $7.58 \%, 0.62 \% \& 2.65 \%$ for PSO and by $5.16 \%, 8.78 \%$ \& $2.5 \%$ for MOPSO when compared with 75,225 $\& 375$ quantity of nodes.

Table 2- Average Packet Delivery Ratio

\begin{tabular}{|l|l|l|l|l|}
\hline Quantity of nodes & LEACH & PSO & MOPSO & MOPSO-DE \\
\hline 75 & 0.720416 & 0.7755 & 0.8711 & 0.892 \\
\hline 150 & 0.68758 & 0.7412 & 0.8473 & 0.8561 \\
\hline 225 & 0.680918 & 0.7122 & 0.8252 & 0.8442 \\
\hline 300 & 0.644517 & 0.6862 & 0.7974 & 0.8044 \\
\hline 375 & 0.593199 & 0.6434 & 0.7214 & 0.7432 \\
\hline 450 & 0.513714 & 0.567 & 0.691 & 0.7121 \\
\hline
\end{tabular}


Multi-hop Wireless Sensor Networks

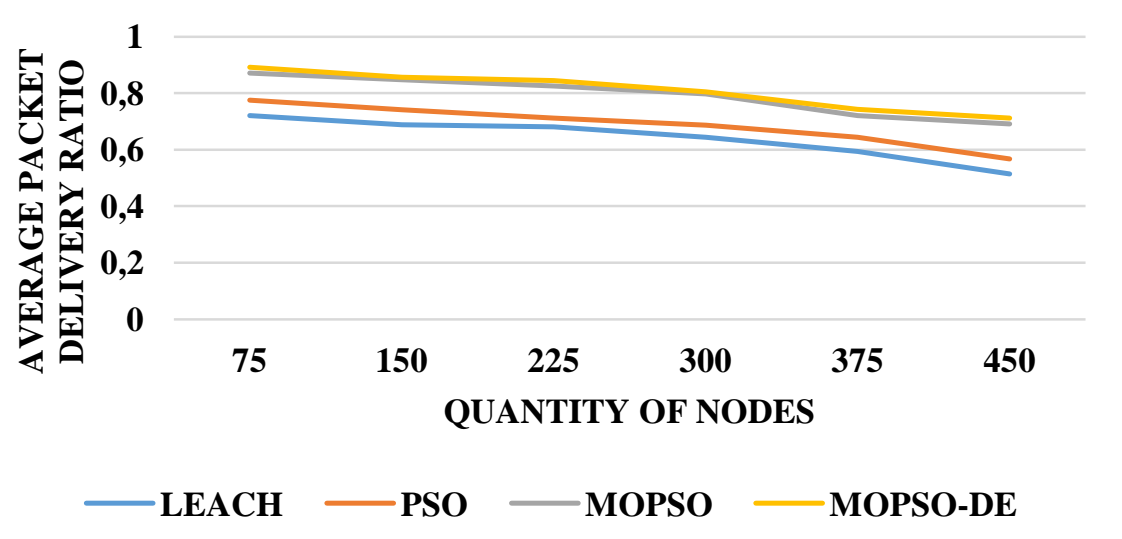

Figure 4 - Average Packet Delivery Ratio

From the figure 4, it is seen that the MOPSO-DE has higher average packet delivery ratio by $21.28 \%, 21.41 \%$ \& $22.44 \%$ for LEACH, by $13.97 \%, 16.96 \%$ \& $14.39 \%$ for PSO and by $2.37 \%, 2.27 \%$ \& $2.97 \%$ for MOPSO when compared with $75,225 \& 375$ quantity of nodes.

Table 3 - Percentage of Nodes Alive

\begin{tabular}{|l|l|l|l|l|}
\hline $\begin{array}{l}\text { Quantity of } \\
\text { rounds }\end{array}$ & LEACH & PSO & MOPSO & MOPSO-DE \\
\hline 0 & 100 & 100 & 100 & 100 \\
\hline 100 & 92 & 95 & 95 & 96 \\
\hline 200 & 74 & 82 & 88 & 90 \\
\hline 300 & 62 & 72 & 78 & 79 \\
\hline 400 & 13 & 32 & 67 & 71 \\
\hline 500 & 0 & 9 & 52 & 54 \\
\hline 600 & 0 & 0 & 9 & 10 \\
\hline 700 & 0 & 0 & 7 & 9 \\
\hline
\end{tabular}

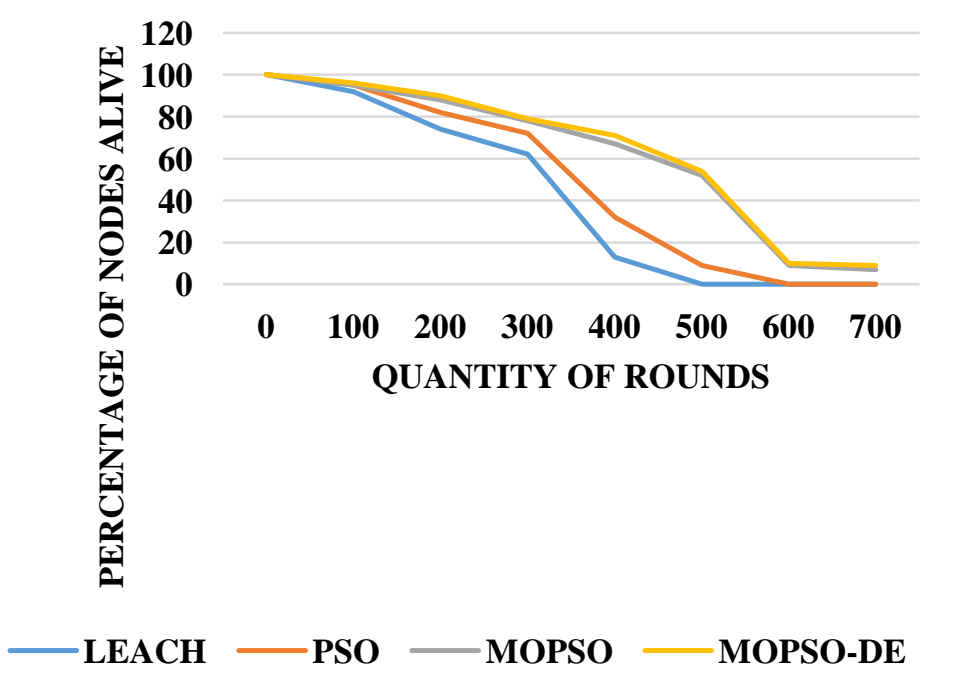

Figure 5 - Percentage of Nodes Alive 


\section{CONCLUSION}

WSNs are a new information technology infrastructure class where computing is embedded into the physical world. WSN applications include building control, environmental monitoring, traffic control, manufacturing, service robotics, and plant automation and surveillance. Clustering is used for energy efficient data communication. This paper proposed a MOPSODE for efficient clustering.

Experiments prove that the new method outperforms MOPSO-DE by improving packet delivery ratio and percentage of nodes alive and by reducing end to end delay. Further investigation to reduce the end to end delay is required.

\section{REFERENCES}

Nguyen, D., Minet, P., Kunz, T., \& Lamont, L. (2011, June). New findings on the complexity of cluster head selection algorithms. In World of Wireless, Mobile and Multimedia Networks (WoWMoM), 2011 IEEE International Symposium on a (pp. 110). IEEE.

Romoozi, M., \& Ebrahimpour-Komleh, H. (2012). A positioning method in wireless sensor networks using genetic algorithms. Physics Procedia, 33, 1042-1049.

He, S., Dai, Y., Zhou, R., \& Zhao, S. (2012). A clustering routing protocol for energy balance of wsn based on genetic clustering algorithm. IERI, Procedia, 2, 788-793.

Singh, B., \& Lobiyal, D. K. (2012). A novel energy-aware cluster head selection based on particle swarm optimization for wireless sensor networks. human-Centric Computing and Information Sciences, 2(1), 1-18.

Kumar, D. (2014). Performance analysis of energy efficient clustering protocols for maximising lifetime of wireless sensor networks. Wireless Sensor Systems, IET, 4(1), 916.

Elhabyan, R. S., \& Yagoub, M. C. (2014, September). Energy efficient clustering protocol for WSN using PSO. In Global Information Infrastructure and Networking Symposium (GIIS), 2014 (pp. 1-3). IEEE.

Maleki, I., Khaze, S. R., Tabrizi, M. M.,\& Bagherinia, A. (2013). A new Tyagi, S., \& Kumar, N. (2013). A new approach for area coverage problem in Wireless Sensor Networks with hybrid particle swarm optimization and differential evolution algorithms. International Journal of Mobile Network Communications and Telematics (IJMNCT), 3(6), 61-76.

Tyagi, S., \& Kumar, N. (2013). A systematic review on clustering and routing techniques based upon LEACH protocol for wireless sensor networks. Journal of Network and Computer Applications, 36(2), 623- 645

Aziz, N. A. A., Mohemmed, A. W., \& Zhang, M. (2010). Particle swarm optimization for coverage maximization and energy conservation in wireless sensor networks. In Applications of Evolutionary Computation (pp. 51-60). Springer Berlin Heidelberg.

Yu, H., \& Xiaohui, W. (2011). PSO-based Energy-balanced Double Cluster-heads Clustering Routing for wireless sensor networks. Procedia Engineering, 15, 3073-3077.

Rostami, A., \& Mottar, M. H. (2014). Wireless Sensor Network Clustering Using Particles Swarm Optimization for Reducing Energy Consumption. International Journal of Managing Information Technology, 6(4). 
Elhabyan, R. S., \& Yagoub, M. C. (2015). Two-tier particle swarm optimization protocol for clustering and routing in wireless sensor network. Journal of Network and Computer Applications, 52, 116-128.

Yadav,R. K., Kumar, V., \& Kumar, R. (2015). A discrete particle swarm optimization based clustering algorithm for wireless sensor networks. In Emerging ICT for Bridging the Future-Proceedings of the 49th Annual Convention of the Computer Society of India CSI Volume 2 (pp. 137-144). Springer International Publishing.

Kennedy, J., \& Eberhart, R. C. (1997, October). A discrete binary version of the particle swarm algorithm. In Systems, Man, and Cybernetics, 1997. Computational Cybernetics and Simulation., 1997 IEEE International Conference on (Vol. 5, pp. 41044108). IEEE.

Kuila, P., \& Jana, P. K. (2014). Energy efficient clustering and routing algorithms for wireless sensor networks: Particle swarm optimization approach. Engineering Applications of Artificial Intelligence, 33, 127-140.

Durillo, J. J., García-Nieto, J., Nebro, A. J., Coello, C. A. C., Luna, F., \& Alba, E. (2009, April). Multi-objective particle swarm optimizers: An experimental comparison. In Evolutionary Multi-Criterion Optimization (pp. 495-509). Springer Berlin Heidelberg.

Li, X., Xu, L., Wang, H., Song, J., \& Yang, S. X. (2010). A differential evolution-based routing algorithm for environmental monitoring wireless sensor networks. Sensors, 10(6), 5425-5442.

Maleki, I., Khaze, S. R., Tabrizi, M. M., \&Bagherinia, A. (2013). A new approach for area coverage problem in Wireless Sensor Networks with hybrid particle swarm optimization and differential evolution algorithms. International Journal of Mobile Network Communications and Telematics (IJMNCT), 3(6), 61-76.

Abraham, A., Jatoth, R. K., \&Rajasekhar, A. (2012). Hybrid differential artificial bee colony algorithm. Journal of Computational and Theoretical Nanoscience,9(2), 249-257.

Wickramasinghe, U., \& Li, X. (2008, December). Choosing leaders for multi-objective PSO algorithms using differential evolution. In Asia-Pacific Conference on Simulated Evolution and Learning (pp. 249-258). Springer Berlin Heidelberg.

Yadav, L., \& Sunitha, C. (2014). Low Energy Adaptive Clustering Hierarchy in Wireless Sensor Network (LEACH). International Journal of Computer Science \& Information Technologies, 5(3). 